\title{
Correction to: The Green Road to Environmental Performance: A Study of Private Banking Sector in Colombo District, Sri Lanka
}

P. N. Sandaruwan, U. K. Thalgaspitiya, and W. N. Hettiarachchi

\section{Correction to:}

Chapter 18 in: S. Vanka et al. (eds.), Sustainable Human Resource Management, https://doi.org/10.1007/978-981-15-5656-2_18

In the original version of the book, the affiliations of authors "P. N. Sandaruwan, U. K. Thalgaspitiya, W. N. Hettiarachchi” have been updated in the chapter 18. The chapter and book have been updated with the changes. 